\title{
SMARCE1 mutations in pediatric clear cell meningioma: case report
}

\author{
Linton T. Evans, MD, ${ }^{1}$ Jack Van Hoff, MD, ${ }^{2,4,5}$ William F. Hickey, MD, ${ }^{3,4}$ Miriam J. Smith, PhD, ${ }^{6}$ \\ D. Gareth Evans, MD, ${ }^{6}$ William G. Newman, MD, PhD, ${ }^{6}$ and David F. Bauer, MD ${ }^{1,2,4,5}$
}

\begin{abstract}
${ }^{1}$ Section of Neurosurgery, ${ }^{2}$ Department of Pediatrics, and ${ }^{3}$ Department of Pathology, Dartmouth-Hitchcock Medical Center; ${ }^{4}$ Norris Cotton Cancer Center; ${ }^{5}$ Children's Hospital at Dartmouth-Hitchcock, Lebanon, New Hampshire; and ${ }^{6}$ Manchester Centre for Genomic Medicine, Manchester Academic Health Sciences Centre (MAHSC), St. Mary's Hospital, University of Manchester, United Kingdom
\end{abstract}

Clear cell meningioma (CCM) is an uncommon variant of meningioma. The authors describe a case of a pediatric CCM localized to the lumbar spine. After resection, sequencing revealed an inactivating mutation in the SWI/SNF chromatin remodeling complex subunit SMARCE1, with loss of the second allele in the tumor. The authors present a literature review of this mutation that is associated with CCM and a family history of spine tumors.

http://thejns.org/doi/abs/10.3171/2015.3.PEDS14417

KEY WORDS clear cell meningioma; SMARCE1; SWI/SNF; oncology

$\mathrm{C}$ LEAR cell meningioma $(\mathrm{CCM})$ is a rare histopathological variant originally described in 1990 . This subtype is estimated to account for less than $1 \%$ of meningiomas but is frequently difficult to treat. Despite a benign histological appearance, these tumors exhibit aggressive behavior with up to $60 \%$ recurring following resection. They are classified accordingly as WHO Grade II tumors. Clear cell meningiomas exhibit a predilection for pediatric patients and typically arise in the lumbosacral spine. . $-6,8,9,11,12,18,21$ The molecular underpinnings of CCM tumorigenesis and progression remain poorly defined. Recently, heterozygous loss-of-function mutations in the SWI/SNF chromatin remodeling complex subunit SMARCE1 were found in 4 individuals with familial multiple spinal meningiomas. ${ }^{15}$ None of these individuals had features of neurofibromatosis Type 2 (NF2), and resected meningiomas were all of the clear cell variant. Mutations in SMARCE1 and other SWI/SNF subunits have been implicated in a number of human tumors. ${ }^{7,19}$ Investigation into the function of this complex has established tumor suppressor properties and defined a role in cell differentiation and proliferation. ${ }^{13,20}$ We describe a further case of a SMARCE1 mutation and loss of heterozygosity in a 3-year-old boy with an intraspinal CCM and a relative with a known spinal tumor.

\section{Case Report}

History and Examination

A 3-year-old boy presented to the neurosurgical service with 6 weeks of bilateral knee pain, refusal to walk, and urinary incontinence. Spontaneous movement of his lower extremities was symmetrical and his strength was normal during manual motor testing in all muscle groups. His sensation was intact to all modalities in his lower extremities, although he had a diminished left patellar deep tendon reflex. There was evidence of urinary retention with approximately 1 liter of urine obtained with insertion of an indwelling Foley catheter. MRI of his spine (Fig. 1) revealed a T1- and T2-isointense lobulated mass measuring $3 \times 1.5$ $\mathrm{cm}$ with homogenous contrast enhancement. This intradural extramedullary mass effaced the CSF space at L1-2 with displacement of the nerve roots. The lesion appeared to abut but not invade the conus medullaris. Based on

ABBREVIATIONS CCM = clear cell meningioma; NF2 = neurofibromatosis Type 2.

SUBMITTED August 12, 2014. ACCEPTED March 18, 2015.

INCLUDE WHEN CITING Published online June 26, 2015; DOI: 10.3171/2015.3.PEDS14417.

DISCLOSURE The authors report no conflict of interest concerning the materials or methods used in this study or the findings specified in this paper. 

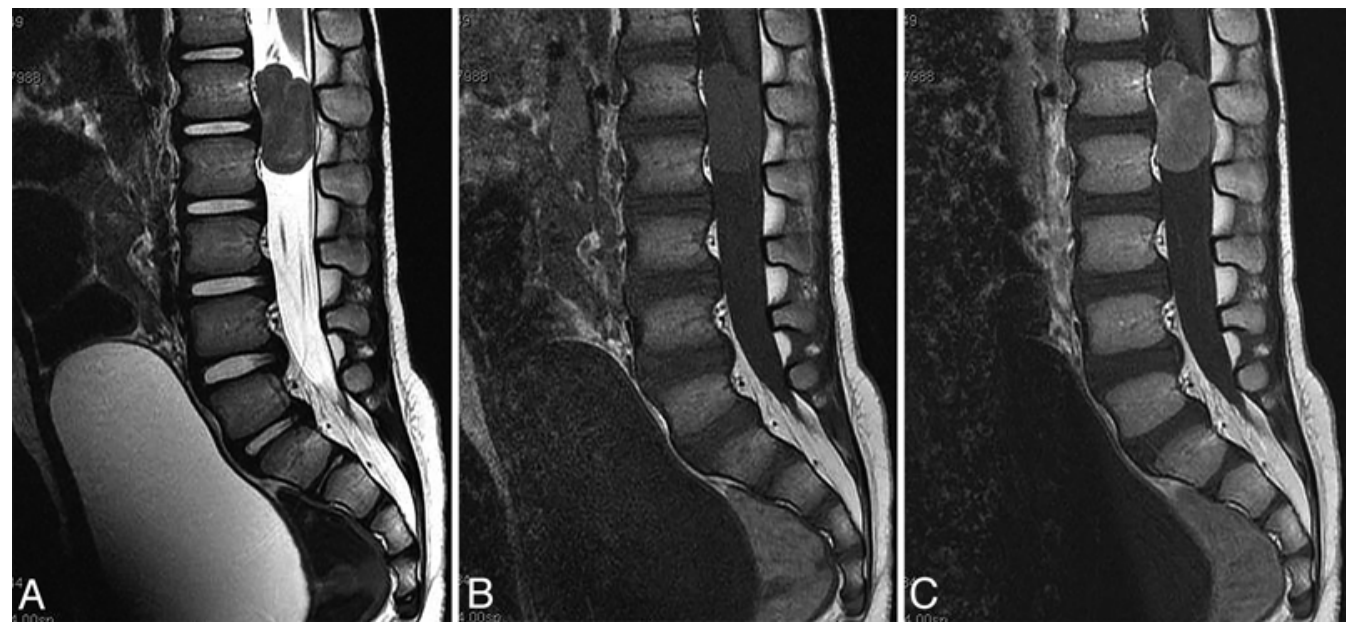

FIG. 1. Preoperative sagittal T2-weighted (A), T1-weighted without Gd (B), and T1-weighted with Gd (C) MR images demonstrating maximal diameter of the isointense intramedullary mass $(A)$ and homogenous enhancement of the described mass $(C)$.

the radiological findings, the tumor differential included nerve sheath tumor, myxopapillary ependymoma, meningioma, lymphoma, and germ cell tumor. The remainder of the neuraxis was subsequently imaged with no additional tumors identified in the brain or other spinal segments.

\section{Operation and Postoperative Course}

Laminectomies at L1-3 were performed with a midline durotomy to expose the tumor. An expansile and opaque mass was immediately identified displacing the surrounding nerve roots. The filum terminale was transected to optimize visualization of the mass and mobilize the conus rostrally. Ultrasonic aspirator and microdissection techniques were used to resect the mass. Gross-total resection of the mass was confirmed radiologically (Fig. 2). Postoperatively the patient was neurologically intact and ambulating without assistance. At the time of discharge his radicular pain had resolved. Normal bladder function was restored by 4 weeks. At the 18-month follow-up evaluation he had no new symptoms, and repeat MRI did not demonstrate any evidence of tumor recurrence or progression. No adjuvant chemotherapy or radiation therapy has been offered to date.

\section{Pathological and Genetic Analysis}

The tumor displayed typical pathological features of CCM. Moderately cellular sheets of polygonal cells with clear cytoplasmic staining were prominent. Glycogen contained within the cytoplasm exhibited characteristic staining with PAS (Fig. 3). Immunohistochemical staining for epithelial membrane antigen was positive, and the Ki 67 labeling index was $20 \%$. Sanger sequencing of SMARCE1 DNA isolated from peripheral circulating lymphocytes revealed a heterozygous insertion of adenine in exon 6 of SMARCE1, c.275_276insA, p.(Leu93Valfs*17). This was predicted to result in a frameshift mutation..$^{15}$ Sequencing of tumor DNA from formalin-fixed tissue specimens detected loss of heterozygosity, noted as a reduced peak height of the wild-type SMARCE1 allele in tumor DNA compared with lymphocyte DNA (Fig. 4). The second wild-type allele was lost in the neoplastic cells. Immuno- histochemical analysis of paraffin-embedded tumor tissue was negative for SMARCE1 protein $^{16}$ relative to control slides. ${ }^{15}$

\section{Family History}

The patient has a paternal uncle with a lumbar spinal tumor that required resection. The uncle was not available for follow-up, and his tumor histology was not available for review.

\section{Discussion}

Clear cell meningiomas are rare tumors. They are most often reported to affect children and frequently involve the lumbar spine. Patients typically present with leg and back pain with bowel or bladder dysfunction. The benign histological appearance is deceptive, with the majority of tumors recurring either locally or at distant sites following gross-total resection. Death associated with CCM is reported to be as high as $23 \%$, with median time to recurrence ranging from approximately 6 to 18 months. ${ }^{4}$ Currently the pathogenesis of these tumors is elusive, and recurrence is difficult to predict. Ki 67 labeling has been offered as a prognostic indicator with an index greater than 3\% predicting recurrence, but few Ki 67 values have been reported in the literature. Notably, Park et al. reported a patient with a CCM and a Ki 67 labeling index of 3\% who experienced a local recurrence. Histopathology was unchanged at the time of recurrence, but the Ki 67 labeling index had increased from 3\% to $20 \% .{ }^{12}$ Oviedo et al. reported a patient with a Ki 67 labeling index of $10 \%$ but no disease progression at 1 year following total resection. ${ }^{11}$ In the case presented here, the Ki 67 labeling index was $20 \%$ with no evidence of local or distant disease at the 18-month follow-up examination. Due to the aggressive nature of these tumors, adjuvant radiotherapy has been used in a number of patients. We chose close follow-up without adjuvant treatment for our patient. Because of the limited data available on radiation therapy after grosstotal resection of spinal CCM, it is unclear what the role for radiotherapy should be in the treatment of CCM. Furthermore, as chromatin remodeling complexes are vital to 


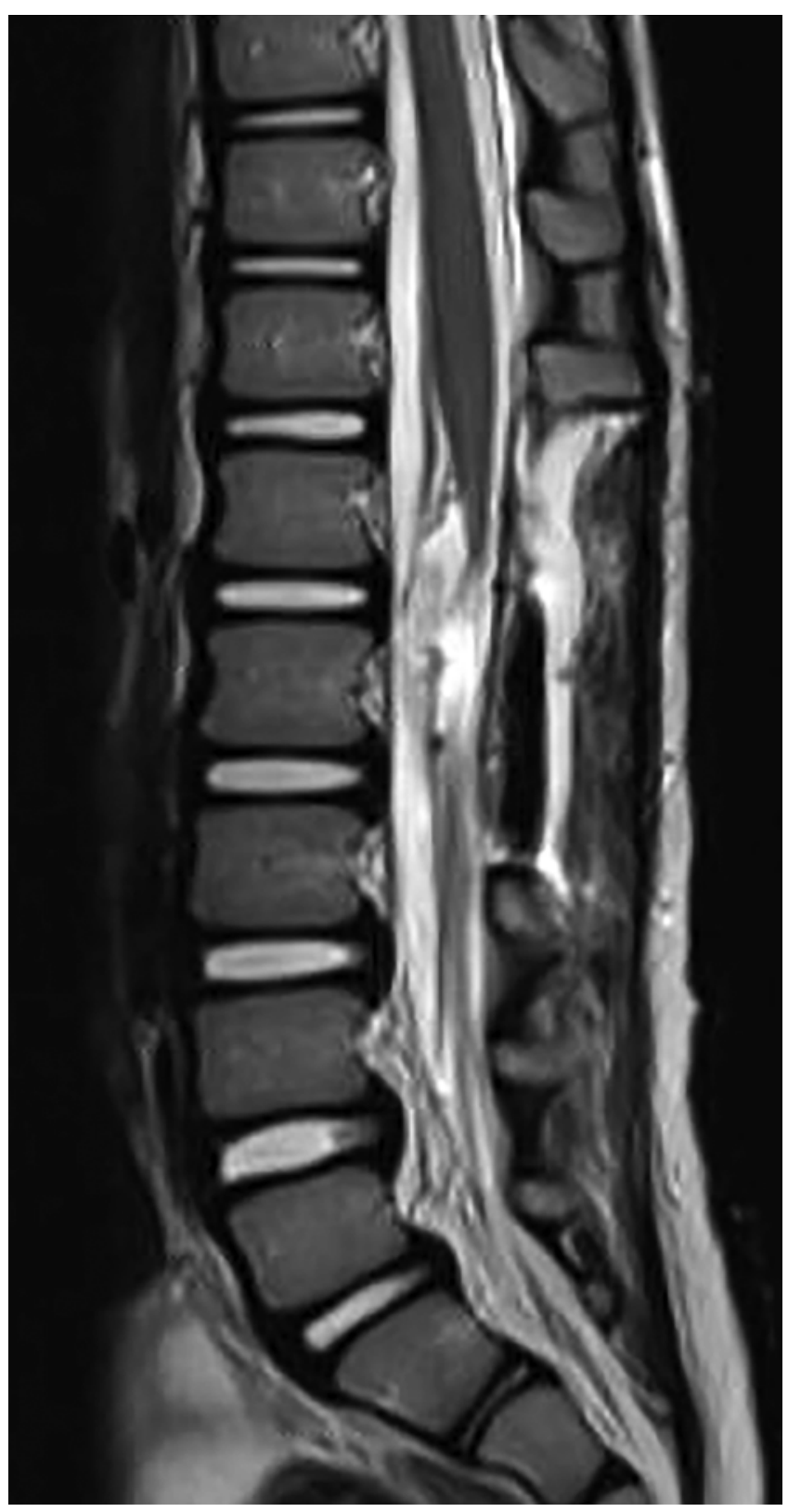

FIG. 2. Postoperative sagittal T2-weighted MR image demonstrating interval resection of the known mass with relief of mass effect on the conus and surrounding nerve roots.

double-stranded DNA repair following ionizing radiation, there is a theoretical risk that patients lacking SMARCE1 may be more sensitive to radiotherapy. ${ }^{10}$ Regardless of the completeness of resection, pathological features such as Ki 67 labeling index, or use of adjuvant therapy, these patients require frequent imaging of the entire neuraxis for early detection of recurrent tumor growth. Recurrence has been documented in as little as 6 months. In our practice, we recommend surveillance MRI at 6-month intervals for 3 years, followed by yearly MRI for an additional 2 years.

A unique syndrome of multiple inherited spinal meningiomas related to mutations in the SWI/SNF chromatin remodeling complex subunit SMARCE1 has been recently described. ${ }^{15}$ Smith et al. identified 4 individuals with
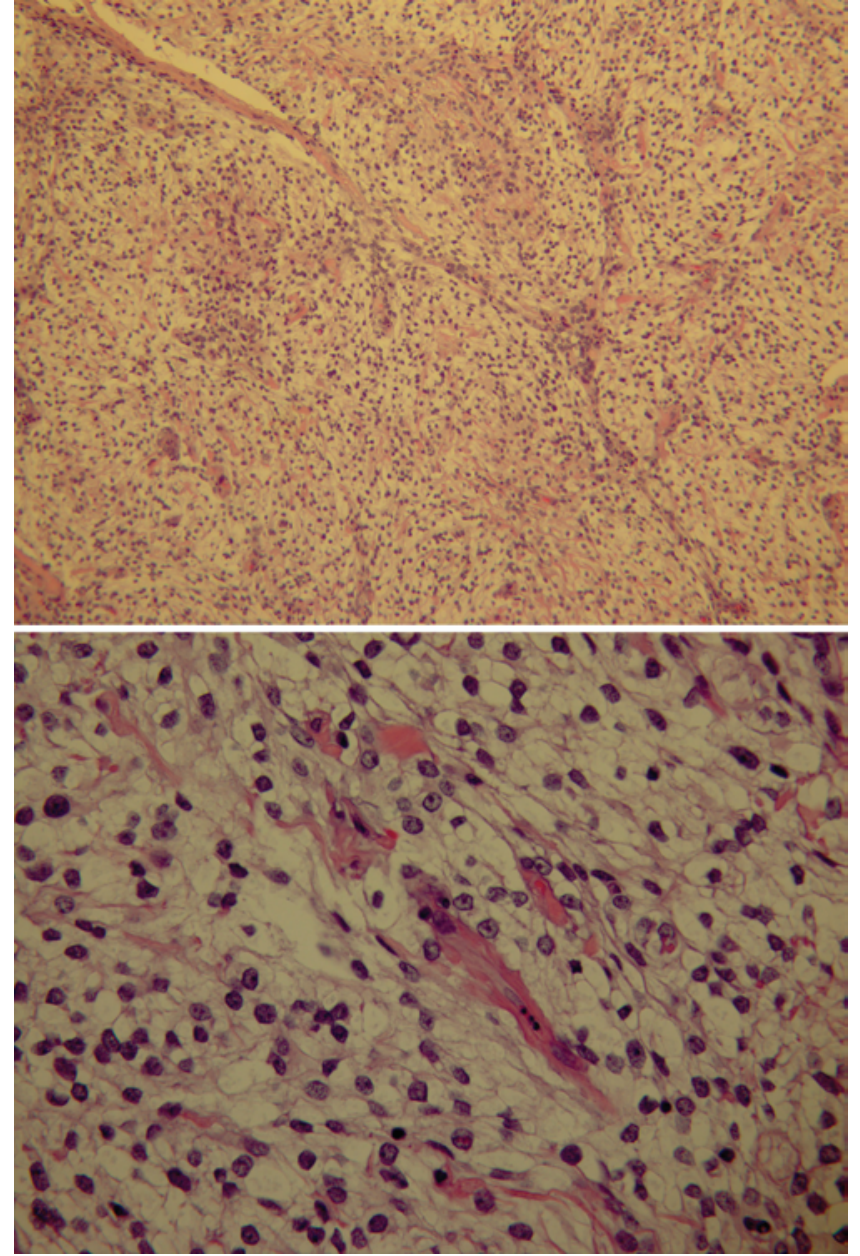

FIG. 3. Photomicrographs demonstrating a moderately cellular tumor composed of polygonal cells with clear cytoplasmic staining consistent with CCM. $H$ \& E, original magnification $\times 100$ (upper) and $\times 400$ (lower). Figure is available in color online only.

spinal CCMs and an affected relative with heterozygous germline mutations in SMARCE1.15 The mutations led to a nonfunctional product, and the tumors exhibited loss of heterozygosity consistent with a tumor suppressor action for SMARCE1. Sequencing of SMARCE1 from circulating lymphocytes in our patient identified a germline mutation. The second wild-type allele was additionally lost in tumor cells. Parental samples were not available for sequence analysis, but because the patient had a paternal uncle with a spinal tumor, the mutation may be paternally inherited, but we are unable to confirm this at this time.

Of the individuals with spinal CCMs in the original report described above, one had loss of the second allele that encompassed NF2. Tumor development in individuals with loss of SMARCE1 appears to occur independently of NF2.

SWI/SNF complexes regulate chromatin structure through an ATP-dependent nucleosome remodeling activity. In addition, they interact with other chromatin remodeling proteins and recruit histone deacetylases. ${ }^{20}$ Inactivating mutations in SWI/SNF subunits have been found in a number of human tumors, suggesting a tumor suppressor 


\section{WT TAAAGGCTTCCAACCCTGACCTAAAGTTGTGGGAGATTGGCAAGATTATTGGTGGCAT mut TAAAGGCTTCCAACCCTGACCTAAAAGTTGTGGGAGATTGGCAAGATTATTGGTGGCA}

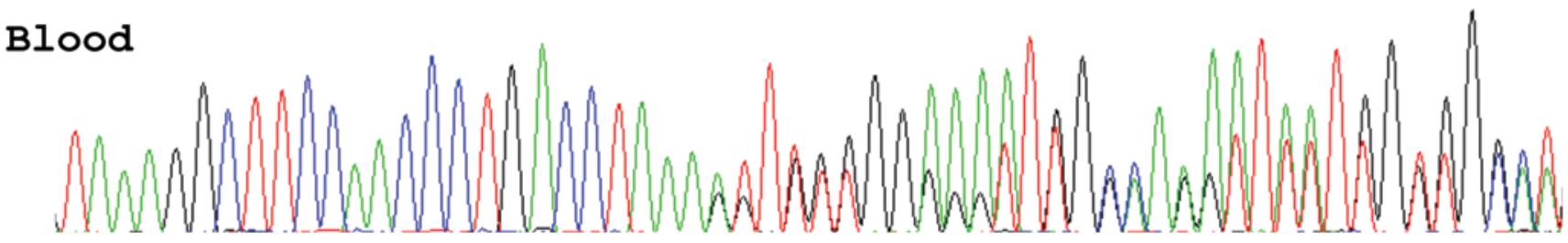

Tumor

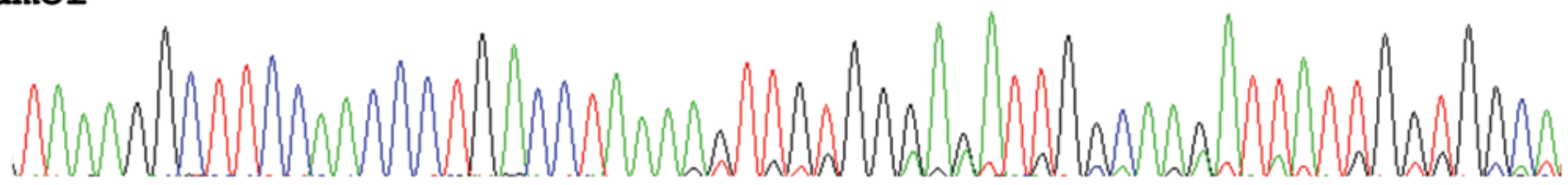

FIG. 4. Sequencing chromatograms for SMARCE1 obtained from circulating lymphocyte and tumor DNA. There is loss of the peak height of the wild-type (WT) allele in tumor but not lymphocyte DNA, indicating loss of the second wild-type allele in tumor cells. Red " $A$ " indicates the mutation (mut). Each color in the chromatogram represents a different DNA base pair. Figure is available in color online only.

function. The SNF5 or SMARCB1 subunit is inactivated in familial rhabdoid tumors and schwannomas, and SNF5 heterozygous mice develop sarcomas with spontaneous loss of the second allele recapitulating human tumors. ${ }^{1,2}$ Homozygous mice rapidly develop lymphomas and rhabdoid tumors..$^{14}$ Somatic mutations in PBRMI are found in up to $40 \%$ of renal cell carcinomas and ARIDIA is mutated in over $50 \%$ of ovarian clear cell carcinomas and rarely medulloblastomas. Somatic variants in the SWI/ SNF complex have also been found in a minority of breast cancers and non-small cell lung carcinoma. ${ }^{20}$

The pathophysiology surrounding loss of function of SWI/SNF complexes is poorly understood and potentially multifactorial depending on the affected subunits. SWI/ SNF complexes are involved in regulating lineage-specific differentiation from neurogenesis to hematopoiesis. SWI/ SNF subunits interact with multiple transcription factors, balancing transcriptional activation of lineage-specific gene products while suppressing other proliferative pathways. Heterozygous loss of the subunit $\mathrm{Brgl}$ led to neural tube defects, reduced brain volumes, and loss of neural progenitor cells in a murine model. Activating germline mutations in SMARCE1 have been described in the human neurodevelopmental disorder Coffin-Siris syndrome, which is characterized by intellectual impairment, microcephaly, coarse facial features, and nail hypoplasia. ${ }^{17}$ Although our patient does not have Coffin-Siris syndrome, it is interesting that he exhibited hypoplasia of the cerebellar vermis.

The finding that loss-of-function mutations in SMARCE1 cause CCMs adds to the emerging evidence of tumor suppressor activity of SWI/SNF complexes. Our understanding of the importance of chromatin remodeling proteins and epigenetic regulators in tumorigenesis, particularly pediatric tumors, is increasing and defines potential therapeutic targets. It is interesting that these tumors are often of clear cell histology. Individuals with CCMs represent a unique subset of patients due to the germline SMARCE1 mutation having implications in the pathogenesis, prognosis, and treatment of these lesions.
Clear cell meningiomas exhibit aggressive behavior with a tendency to recur following resection. Tumors are commonly located in the spine and affect young individuals. The pathogenesis of these tumors is not well defined. The patient reported in this paper was found to have a germline mutation in SMARCE1, a subunit in the SWI/ SNF chromatin remodeling complex, with loss of heterozygosity in the tumor. Loss-of-function mutations have been reported in SMARCE1 in individuals with spinal CCMs, defining a unique familial tumor syndrome. Further studies in SMARCEl will be important to understand the development of these tumors and the role of chromatin remodeling proteins in tumorigenesis, and will help to identify individuals at risk for multiple meningiomas.

\section{References}

1. Biegel JA, Kalpana G, Knudsen ES, Packer RJ, Roberts CW, Thiele CJ, et al: The role of INI1 and the SWI/SNF complex in the development of rhabdoid tumors: meeting summary from the workshop on childhood atypical teratoid/rhabdoid tumors. Cancer Res 62:323-328, 2002

2. Biegel JA, Zhou JY, Rorke LB, Stenstrom C, Wainwright LM, Fogelgren B: Germ-line and acquired mutations of INI1 in atypical teratoid and rhabdoid tumors. Cancer Res 59:74-79, 1999

3. Carrà S, Drigo P, Gardiman M, Perilongo G, Rigobello L: Clear-cell meningioma in a 22-month-old male: a case report and literature review. Pediatr Neurosurg 34:264-267, 2001

4. Colen CB, Rayes M, McClendon J Jr, Rabah R, Ham SD: Pediatric spinal clear cell meningioma. Case report. J Neurosurg Pediatr 3:57-60, 2009

5. Heth JA, Kirby P, Menezes AH: Intraspinal familial clear cell meningioma in a mother and child. Case report. J Neurosurg 93 (2 Suppl):317-321, 2000

6. Jia Y, Zhong DR, Cui QC: Intraspinal clear cell meningioma: a case report. Chin Med J (Engl) 118:348-349, 2005

7. Kiskinis E, García-Pedrero JM, Villaronga MA, Parker MG, Belandia B: Identification of BAF57 mutations in human breast cancer cell lines. Breast Cancer Res Treat 98:191198, 2006

8. Liu PI, Liu GC, Tsai KB, Lin CL, Hsu JS: Intraspinal clearcell meningioma: case report and review of literature. Surg Neurol 63:285-289, 2005 
9. Miranda P, Simal JA, Vila M, Hernández M, Menor F, Alvarez-Garijo JA: Posterior fossa clear cell meningioma without dural attachment in a child. Childs Nerv Syst 25:389-392, 2009

10. Ogiwara H, Ui A, Otsuka A, Satoh H, Yokomi I, Nakajima S, et al: Histone acetylation by CBP and p300 at double-strand break sites facilitates SWI/SNF chromatin remodeling and the recruitment of non-homologous end joining factors. Oncogene 30:2135-2146, 2011

11. Oviedo A, Pang D, Zovickian J, Smith M: Clear cell meningioma: case report and review of the literature. Pediatr Dev Pathol 8:386-390, 2005

12. Park HC, Sohn MJ, Kim EY, Han HS, Park HS: Spinal clear cell meningioma presented with progressive paraparesis in infancy. Childs Nerv Syst 16:607-610, 2000

13. Reisman D, Glaros S, Thompson EA: The SWI/SNF complex and cancer. Oncogene 28:1653-1668, 2009

14. Roberts CW, Leroux MM, Fleming MD, Orkin SH: Highly penetrant, rapid tumorigenesis through conditional inversion of the tumor suppressor gene Snf5. Cancer Cell 2:415-425, 2002

15. Smith MJ, O'Sullivan J, Bhaskar SS, Hadfield KD, Poke $\mathrm{G}$, Caird J, et al: Loss-of-function mutations in SMARCE1 cause an inherited disorder of multiple spinal meningiomas. Nat Genet 45:295-298, 2013

16. Smith MJ, Wallace AJ, Bennett C, Hasselblatt M, Elert-Dobkowska E, Evans LT, et al: Germline SMARCE1 mutations predispose to both spinal and cranial clear cell meningiomas. J Pathol 234:436-440, 2014

17. Tsurusaki Y, Okamoto N, Ohashi H, Kosho T, Imai Y, Hibi-
Ko Y, et al: Mutations affecting components of the SWI/SNF complex cause Coffin-Siris syndrome. Nat Genet 44:376378, 2012

18. Vural M, Arslantaş A, Ciftçi E, Artan S, Atasoy MA: An unusual case of cervical clear-cell meningioma in pediatric age. Childs Nerv Syst 23:225-229, 2007

19. Wang L, Baiocchi RA, Pal S, Mosialos G, Caligiuri M, Sif S: The BRG1- and hBRM-associated factor BAF57 induces apoptosis by stimulating expression of the cylindromatosis tumor suppressor gene. Mol Cell Biol 25:7953-7965, 2005

20. Wilson BG, Roberts CW: SWI/SNF nucleosome remodellers and cancer. Nat Rev Cancer 11:481-492, 2011

21. Zorludemir S, Scheithauer BW, Hirose T, Van Houten C, Miller G, Meyer FB: Clear cell meningioma. A clinicopathologic study of a potentially aggressive variant of meningioma. Am J Surg Pathol 19:493-505, 1995

\section{Author Contributions}

Conception and design: Bauer. Acquisition of data: Smith, DG Evans, Newman. Analysis and interpretation of data: Smith, Newman. Drafting the article: Bauer, LT Evans, Van Hoff, DG Evans, Newman. Critically revising the article: all authors. Reviewed submitted version of manuscript: all authors. Prepared pathology figures and slides: Hickey.

\section{Correspondence}

David F. Bauer, Pediatric Neurosurgery, Dartmouth-Hitchcock Medical Center, One Medical Center Dr., Lebanon, NH 03756. email: david.f.bauer@hitchcock.org. 